\title{
Effects of an Educational Program of Physical Exercise and Nutrition in Overweight or Obese Adults Suffering from Binge Eating Disorder
}

\author{
A. Barroco ${ }^{1}$, D. Collado-Mateo ${ }^{2,3}$, S. Villafaina ${ }^{2,3}$, J. Bravo ${ }^{1,4}$ and J. A. Parraca ${ }^{1,4}$ \\ 1. Departamento de Desporto e Saúde, Escola de Ciência e Tecnologia, Universidade de Évora, Évora, Portugal \\ 2. Physical Activity and Quality of Life Research Group (AFYCAV), Faculty of Sport Sciences, University of Extremadura, Cáceres, \\ Spain \\ 3. Facultad de Educacion, Universidad Autonoma de Chile, Talca, Chile \\ 4. Comprehensive Health Research Center (CHRC), University of Évora, Portugal
}

\begin{abstract}
Objective: To determine the effects of an educational program of exercise and nutrition in overweight or obese binge eating adults on body composition, physical fitness and biochemical parameters. Being educational, the program also aimed to promote learning and self-control in the practice of physical activity and in the food choices of this population. Methodology: 40 patients overweight or obese binge eating adults aged between 30 and 60 years participated in the study. Participants were assigned to an experimental group (EG) $(N=23)$, to which the program was applied or a control group (CG) $(N=17)$. The study lasted eight months, where an initial and final evaluation was carried out, in order to verify the benefits obtained through this program. The program consisted of 47 one-hour, twice-a-week group practice sessions, one weekly self-help session, and three nutritional monitoring sessions throughout the program. Results: There were significant improvements in the experimental group in the body composition, namely in the percentage of fat mass, fat free mass, fat mass in the trunk, visceral index and metabolic age. In physical fitness, improvements were also seen in the experimental group, specifically in flexibility and handgrip strength. Conclusion: A multidisciplinary program involving regular physical exercise and nutritional educationled to achieve improvements in physical fitness and body composition in overweight and obese binge-eating disorder patients.
\end{abstract}

Key words: Physical exercise, nutrition, obesity, physical fitness, body composition, food compulsion.

\section{Introduction}

Binge eating disorder (BED) consists of frequent and recurrent episodes of binge eating, including rapid eating without being hungry or until being uncomfortable, and the absence of other compensatory behaviors such as vomiting, purging, fasting, excessive exercise or laxative abuse [1]. Among others, it is associated to overweight and obesity, as well as to depression or guilty feelings and other psychiatric disorders [2]. The prevalence of this

Corresponding author: José Alberto Parraca, $\mathrm{PhD}$, research fields: physiology, sports science, management of training programs in special populations. ID-ORCID = 0000-0002-5254-7409. disorder oscillates between 2 and 5\% in general population, being more usual among female with ratio 2:3, i.e. 2 men per 3 women. In Portugal, highest prevalence was observed between 40 and 49 years while those people aged $>65$ would have the lowest risk [3].

BED treatments are focused in the reduction of the frequency of binge-eating events and also in the improvement of the metabolic health and weight control in the case of obese or overweight patients [2]. Common BED treatments can be divided into pharmacological and non-pharmacological approaches. Pharmacological treatments often include antidepressants, anticonvulsants, antiobesity agents 
and central nervous system stimulants. However, most studies evaluating pharmacological treatments report moderate to severe adverse effects including sleep disturbance, headaches, gastrointestinal upset, as well as other well-known side effects that were previously observed in non-BED populations. On the other hand, among non-pharmacological approaches, psychological and behavioral interventions stand as the most successful treatments. These interventions include cognitive behavioral therapies, dialectical behavioral therapy, interpersonal psychotherapy and behavioral weight loss. The combination of physical exercise and dietary therapy has emerged as an alternative therapy to reduce BED symptoms [4].

Apart from the potential improvements in the BED symptoms, physical exercise and nutrition are especially recommended given the benefits in weight and stress control. Guided physical exercise may reduce the onset of negative emotions [5] and may be even as effective as cognitive behavioral therapy to reduce BED symptoms [6]. However, exercise abuse may also be a compensatory strategy for binge eating and that could be the reason why it traditionally has not been prescribed in clinics [7].

Given all the previously mentioned potential benefits of physical exercise, the aim of the current study was to evaluate the effects of a physical exercise and nutritional therapy on weight, body composition, metabolic rate and physical fitness in overweight or obese BED adults.

\section{Methods}

\subsection{Participants}

A total of 40 BED patients participated in this controlled trial, being 30 of them women. The mean age of participants was 47 and mean body mass index was 34. They were derived from their general practitioners, were appropriately informed and signed the written informed consent.

Inclusion criteria include: (a) being aged between 30 and 60 years, and (b) having a body mass index >
$25 \mathrm{~kg} / \mathrm{m}^{2}$. On the other hand, the following exclusion criteria were set: (a) being suffering from cancer, neuromuscular or neurodegenerative disorders, (b) having musculoskeletal injuries that may impair physical exercise and (c) having any other condition that may threat the safe practice of physical activity.

\subsection{Procedure}

Although 45 people were initially invited to participate, only 40 finally agreed to participate. Of these, 23 were allocated to the exercise group and 17 to the control group. The intervention consisted of 8-month physical exercise performed twice a week with a duration of 60 minutes each one. These sessions were conducted in the University and local high school indoor and outdoor sport facilities. Each session included 10 minutes of warm-up, 45 minutes of combined aerobic and strength training, and 5 minutes of walking and stretching.

Apart from these two weekly sessions, participants were encouraged to adopt an active lifestyle and were educated to use technologies to motivate regular exercise like walking. This task was carried out by nurses from the healthcare centers and physical exercise experts. Participants also received one initial session of psychological assistance in groups, which was conducted by a psychologist who collaborated with the project. Furthermore, nutritional education was individually conducted by an expert in the field. On the other hand, the control group continued their usual care, without interrupting their current treatments.

\subsection{Instruments}

Body composition analysis for all participants was performed using the Tanita MC-780 (Tanita Corp, Tokyo, Japan). This device, based on bioelectrical impedance analysis, allows determining the fat mass, fat-free mass, visceral fat index, basal metabolism and metabolic age [8].

Furthermore, physical condition was evaluated 


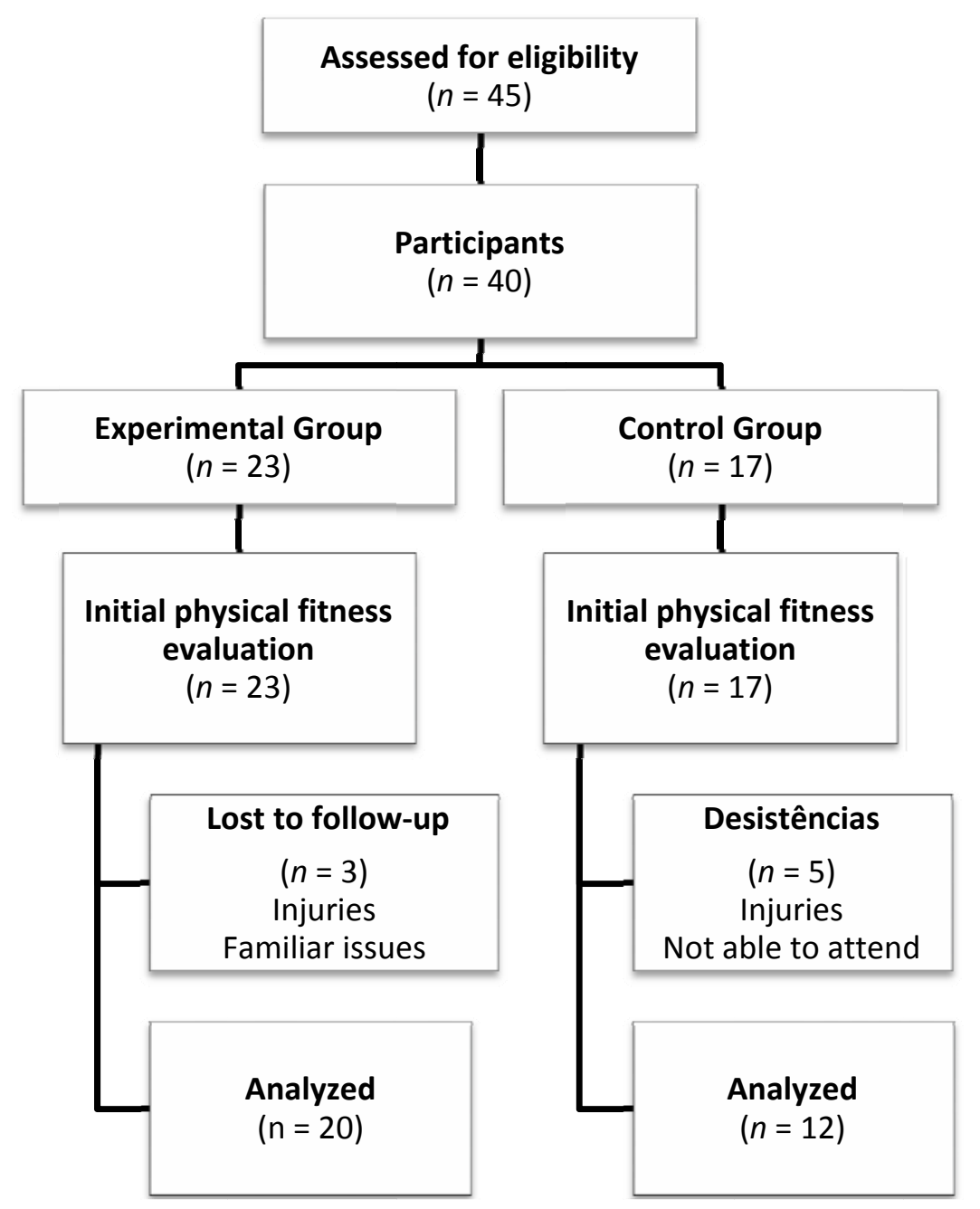

Fig. 1 Flow chart of participants.

before and after the intervention using the 6-min walking test as simple measure of aerobic exercise capacity [9]. This test has been previously used in BED populations [10, 11]. Furthermore, upper extremities strength was evaluated using a handgrip dynamometer (TKK 5101 Grip-D; Takei, Tokyo Japan). The value of the dominant hand was chosen for the statistical analysis.

Moreover, to evaluate flexibility, two tests of the senior fitness test protocol were used [12]: the chair sit and reach and the back scratch test. (1) The chair sit and reach evaluated the lower body flexibility. Participants have to sit at the edge of a chair, extending one leg forward. The distance between the tip of the fingertips and the toes was measured. For the analysis, the mean of left and right arms was used. (2) The back scratch test evaluated the upper body flexibility, measuring how close the hands can be brought together behind the back. Both, left and right values were included in the statistical analysis.

\section{Statistical Analysis}

All statistical analyses were performed using the Statistical Package for Social Sciences (SPSS), version 22. Significance level was set at 0.05 .

Kolmogorov-Smirnoff and Shapiro-Wilk tests were used to determine the use of parametric or non-parametric statistics. A repeated measure ANOVA was performed to compare the effects of the intervention between the exercise and the control 
groups. The interaction (time*group) results were reported.

\section{Results}

Significant improvements were observed in body composition. Specifically, the overall fat mass percentage, trunk fat percentage, fat free mass and visceral fat index were improved after the intervention ( $p$-value $<0.05)$. Weight did not significantly change (see Table 1). Basal metabolism was significantly increased while the metabolic age was reduced in the exercise group after the 8 months intervention.

Regarding physical fitness, handgrip strength and flexibility (upper and lower extremities) were improved ( $p$-value $p<0.05$ ) after the 8-month intervention (see Table 2). On the other hand, there were no significantly differences in cardiovascular according to the ANOVA results. In this regard, the distance covered in the 6-min walking test was increased in more than $10 \%$ in the exercise group while the control group covered $4.27 \%$ more distance after treatment.

Table 1 Effects of the intervention program in the anthropometric measures.

\begin{tabular}{|c|c|c|c|c|}
\hline Variables & Group & Mean at baseline & $\begin{array}{l}\text { Final } \\
\text { mean (SD) }\end{array}$ & $p$-ANOVA \\
\hline \multirow{2}{*}{ Weight (kg) } & EG & $92.23(12.7)$ & $88.9(13.8)$ & \multirow{2}{*}{0.056} \\
\hline & CG & $95.6(17.7)$ & $95.5(18.5)$ & \\
\hline \multirow{2}{*}{$\% \mathrm{FM}$} & EG & $40.8(6.5)$ & $37.4(7.1)$ & \multirow{2}{*}{$0.000 * * *$} \\
\hline & CG & 34.5 (5.3) & $36.2(6.4)$ & \\
\hline \multirow{2}{*}{ \% FM Trunk } & EG & $36.0(4.9)$ & $32.1(4.9)$ & \multirow{2}{*}{$0.000 * * *$} \\
\hline & CG & $33.0(5.2)$ & $35.0(5.5)$ & \\
\hline \multirow{2}{*}{$\%$ FFM } & EG & $60.0(6.4)$ & $62.26(7.6)$ & \multirow{2}{*}{$0.001 * * *$} \\
\hline & CG & $65.6(5.3)$ & $63.8(6.4)$ & \\
\hline \multirow{2}{*}{ Visceral fat index } & EG & $12(3.4)$ & $11(4.7)$ & \multirow{2}{*}{$0.000 * * *$} \\
\hline & CG & $11(4.7)$ & $12(4.8)$ & \\
\hline \multirow{2}{*}{ Basal metabolism (kcal) } & EG & 1694 (215) & 1718 (217) & \multirow{2}{*}{$0.017^{*}$} \\
\hline & CG & $1870(290)$ & $1829(411)$ & \\
\hline \multirow{2}{*}{ Metabolic age (years) } & EG & $60(9.4)$ & $56(7.9)$ & \multirow{2}{*}{$0.024 *$} \\
\hline & CG & $55(6.7)$ & $57(6.7)$ & \\
\hline
\end{tabular}

SD: Standard deviation; \%FM: Percentage of fat mass; \%FFM: Percentage of free fat mass; CE: Experimental Group; CG: Control Group.

$* p<0.05 ; * * p<0.01 ; * * * p<0.001$.

Table 2 Fitness effects of the intervention program.

\begin{tabular}{lllll}
\hline Variables & Group & Mean (SD) at baseline & Mean (SD) after intervention & $p$-ANOVA \\
\hline \multirow{2}{*}{ 6-min walk (m) } & EG & $513.2(44.0)$ & $568.1(53.4)$ & 0.243 \\
& CG & $548.6(100.6)$ & $572(87.2)$ & $0.000^{* * *}$ \\
\multirow{2}{*}{ Arm strength (kg) } & EG & $25.5(8.9)$ & $30.8(7.7)$ & $0.006^{* *}$ \\
\hline \multirow{2}{*}{ Right shoulder flexibility (cm) } & EG & $36.1(16.3)$ & $-5.2(7.1)$ & $0.000^{* * *}$ \\
\hline \multirow{2}{*}{ Left shoulder flexibility (cm) } & EG & $-8.8(8.8)$ & $-10.3(8.8)$ & $-8.7(6.8)$ \\
\hline \multirow{2}{*}{ Trunk flexibility (cm) } & CG & $-8.9(9.6)$ & $-15.2(6.8)$ & $0.002^{* *}$ \\
\hline
\end{tabular}

SD = Standard deviation; CE: Experimental Group; CG: Control Group.

$* p<0.05 ; * * p<0.01 ; * * * p<0.001$. 


\section{Discussion}

The main finding of the current study was that 8-month regular physical activity, along with nutritional education and psychological assistance, significantly improved body composition and physical function in obese or overweight adults suffering from BED. Although changes in weight did not reach statistical significance, the results in the current study are in line with previous research that observed an improvement in physical function and body size and composition [13].

Although the potential benefits of physical exercise in the general population are well known, according to two previous systematic reviews [13, 14], the knowledge about the long-term influence of physical activity in BED is still limited. Comparing our results with those observed in previous studies in BED patients and general population, the non-significant differences in weight and cardiovascular fitness assessed using the 6-min walking test were unexpected. However, another previous study also observed a non-significant between-group improvement in the distance covered in the 6-min walking test [15]. In this regard, a six-month weekly multidisciplinary program, which included regular physical exercise and was conducted in a hospital, achieved an increment close to $20 \%$, which is almost twice the enhancement observed in the current study. That program consisted of 4 weekly sessions of 90 min of aerobic exercise which included activities such as the performance in the 6-min walking test, i.e. brisk walking. Thus, the number of weekly minutes of aerobic physical exercise was largely higher in that study compared with the current study, which could potentially explain the results observed in both studies.

Apart from the aerobic component of physical function and exercise, the exercise program of the current study also included strength training. This type of exercise is strongly recommended since lower levels of strength are related to loss in functional independence and frailty processes [16]. In this regard, handgrip strength stands as one of the most relevant physical function outcomes, since a reduced score in that test has been associated with an increased risk of many types of mortality, thus it is one of the most widely recommended physical assessment tools in adult populations [17]. Weak hand grip strength in later life is a risk factor for disability, morbidity and mortality and is central to the definitions of sarcopenia and frailty [18, 19]. The current study observed a significant improvement in the handgrip strength of participants from the exercise group compared with those from the control group. This enhancement, along with the reduction in the fat mass, may have led to a reduction in the risk of sarcopenia, mortality and functional dependence.

The current study has several limitations, first, as community program, all participants were not randomly allocated in groups. Second, the sample size did not allow us to compare the effects according to subgroups based on relevant variables such as the type of the obesity, the genre of the patient or the previous level of physical activity. Lastly, the actual diet that participants followed at their homes could not be controlled.

Despite these three limitations, the current study contributes to the understanding of the benefits of physical exercise in BED adults and reports results that are in line with the previous research focused on the effects of multidisciplinary programs in this population.

\section{Conclusion}

A multidisciplinary program based on nutrition and physical exercise is effective at increasing physical function and improving body composition. Improvements in the handgrip strength, along with the reduction of the fat mass in the trunk, may reduce the risk of having a cardiovascular accident and the all-causes risk of dying. According to our results and 
those observed in previous research, physical exercise must be an essential part of multidisciplinary programs aimed to increase the health status of BED patients. The combination of strength training and aerobic exercise may lead to further benefits than aerobic-only physical exercise interventions.

\section{Funding}

UÉvora-UniverCIDADE IV program. (Instituto Português do Desporto e Juventude, I.P., Apoio à AtividadeDesportiva 2018, Contrato-Programa de DesenvolvimentoDesportivo No. CP/605/DD/2018). The author SV was supported by a grant from the regional department of economy and infrastructure of the Government of Extremadura and the European Social Fund (PD16008). The funders played no role in the study design, the data collection and analysis, the decision to publish, or the preparation of the manuscript.

\section{Acknowledgments}

Acknowledgments to Dr. Nuno Pascoa (USF Planicie) and Dr. Vanda Silva (USF Lusitania), Évora, Portugal.

\section{References}

[1] De Zwaan, M. 2001. "Binge Eating Disorder and Obesity.” Int J Obes Relat Metab Disord 25 (Suppl 1): S51-5. doi:10.1038/sj.ijo.0801699.

[2] Brownley, K. A., Berkman, N. D., Peat, C. M., Lohr, K. N., Cullen, K. E., Bann, C. M., and Bulik, C. M. 2016. "Binge-Eating Disorder in Adults: A Systematic Review and Meta-Analysis.” Ann Intern Med 165 (6): 409-20. doi:10.7326/m15-2455.

[3] Pereira, F., Pereira, A. M. G. R., and Veiga-Branco, A. 2017. "Prevalência da compulsão alimentar periódica em adultos.” Acta Portuguesa de Nutrição 9: 50.

[4] Bakland, M., Rosenvinge, J. H., Wynn, R., Sundgot-Borgen, J., Fostervold Mathisen, T., Liabo, K., et al. 2019. "Patients' Views on a New Treatment for Bulimia Nervosa and Binge Eating Disorder Combining Physical Exercise and Dietary Therapy (the PED-t): A Qualitative Study.” Eat Disord, 1-18. doi:10.1080/10640266.2018.1560847.

[5] Bratland-Sanda, S., Martinsen, E. W., Rosenvinge, J. H., Ro, O., Hoffart, A., and Sundgot-Borgen, J. 2011.
"Exercise Dependence Score in Patients with Longstanding Eating Disorders and Controls: The Importance of Affect Regulation and Physical Activity Intensity.” Eur Eat Disord Rev. 19 (3): 249-55. doi:10.1002/erv.971.

[6] Sundgot-Borgen, J., Rosenvinge, J. H., Bahr, R., and Schneider, L. S. 2002. “The Effect of Exercise, Cognitive Therapy, and Nutritional Counseling in Treating Bulimia Nervosa.” Med Sci Sports Exerc 34 (2): 190-5.

[7] Bratland-Sanda, S., Rosenvinge, J. H., Vrabel, K. A., Norring, C., Sundgot-Borgen, J., Ro, O., and Martinsen, E. W. 2009. "Physical Activity in Treatment Units for Eating Disorders: Clinical Practice and Attitudes.” Eat Weight Disord 14 (2-3): e106-12.

[8] Verney, J., Schwartz, C., Amiche, S., Pereira, B., and Thivel, D. 2015. "Comparisons of a Multi-frequency Bioelectrical Impedance Analysis to the Dual-Energy X-Ray Absorptiometry Scan in Healthy Young Adults Depending on their Physical Activity Level.” J Hum Kinet 47: 73-80. doi:10.1515/hukin-2015-0063.

[9] Enright, P. L. 2003. “The Six-Minute Walk Test.” Respir Care 48 (8): 783-5.

[10] Vancampfort, D., De Herdt, A., Vanderlinden, J., Lannoo, M., Adriaens, A., De Hert, M., et al. 2015. "The Functional Exercise Capacity and Its Correlates in Obese Treatment-Seeking People with Binge Eating Disorder: An Exploratory Study.” Disabil Rehabil 37 (9): 777-82. doi:10.3109/09638288.2014.942000.

[11] Vancampfort, D., De Herdt, A., Vanderlinden, J., Lannoo, M., Soundy, A., Pieters, G., et al. 2014. "Health Related Quality of Life, Physical Fitness and Physical Activity Participation in Treatment-Seeking Obese Persons with and without Binge Eating Disorder.” Psychiatry Res 216 (1): 97-102. doi:10.1016/j.psychres.2014.01.015.

[12] Rikli, R. E., and Jones, C. J. 2013. Senior Fitness Test Manual. Human Kinetics.

[13] Blanchet, C., Mathieu, M.-È., St-Laurent, A., Fecteau, S., St-Amour, N., and Drapeau, V. 2018. “A Systematic Review of Physical Activity Interventions in Individuals with Binge Eating Disorders.” Current Obesity Reports 7 (1): 76-88. doi:10.1007/s13679-018-0295-x.

[14] Vancampfort, D., Vanderlinden, J., De Hert, M., Adamkova, M., Skjaerven, L. H., Catalan-Matamoros, D., et al. 2013. "A Systematic Review on Physical Therapy Interventions for Patients with Binge Eating Disorder." Disabil Rehabil $35 \quad$ (26): $2191-6$. doi:10.3109/09638288.2013.771707.

[15] Galasso, L., Montaruli, A., Bruno, E., Pesenti, C., Erzegovesi, S., Cè, E., et al. 2018. “Aerobic Exercise Training Improves Physical Performance of Patients with Binge-Eating Disorder.” Sport Sciences for Health 14 (1): 47-51. doi:10.1007/s11332-017-0398-x. 


\section{Obese Adults Suffering from Binge Eating Disorder}

[16] Fried, L. P., Tangen, C. M., Walston, J., Newman, A. B., Hirsch, C., Gottdiener, J., et al. 2001. "Frailty in Older Adults: Evidence for a Phenotype.” J Gerontol A Biol Sci Med Sci 56 (3): M146-56.

[17] Leong, D. P., Teo, K. K., Rangarajan, S., Lopez-Jaramillo, P., Avezum, A., Jr., Orlandini, A., et al. 2015. "Prognostic Value of Grip Strength: Findings from the Prospective Urban Rural Epidemiology (PURE) Study.”
Lancet 386 (9990):
266-73.

[18] Leong, D. P., and Teo, K. K. 2015. "Predicting Cardiovascular Disease from Handgrip Strength: The Potential Clinical Implications.” Expert Rev Cardiovasc $\begin{array}{lll}\text { Ther } & 13 & \text { (12): }\end{array}$ doi:10.1586/14779072.2015.1101342.

[19] Syddall, H. E., Westbury, L. D., Dodds, R., Dennison, E., Cooper, C., and Sayer, A. A. 2017. "Mortality in the Hertfordshire Ageing Study: Association with Level and Loss of Hand Grip Strength in Later Life.” Age Ageing 46 (3): 407-12. doi:10.1093/ageing/afw222. 